\title{
Principais sensibilizantes em pacientes idosos e não idosos com dermatite de contato
}

\author{
Main allergens in elderly and non-elderly patients with contact dermatitis
}

\author{
Zamir Calamita ${ }^{1} \bowtie$, Andrea Bronhara Pelá Calamita ${ }^{2}$
}

1 Disciplina de Alergia e Imunopatologia Clínica do Programa de Mestrado Acadêmico Saúde e Envelhecimento, Faculdade de Medicina de Marília (FAMEMA). Marília, SP.

2 Disciplina de Dermatologia da FAMEMA. Marília, SP.

\section{RESUMO}

Objetivos: Avaliar a prevalência dos principais sensibilizantes da dermatite de contato alérgica em pacientes idosos e não idosos.

Métodos: Estudo retrospectivo com revisão dos prontuários de pacientes com diagnóstico clínico de dermatite de contato, atendidos no período de agosto de 2000 a janeiro de 2012 no Ambulatório de Alergia da Faculdade de Medicina de Marília e em clínica particular de Alergia e Dermatologia na cidade de Marília, estado de São Paulo. Os critérios de inclusão foram diagnóstico clínico de dermatite de contato, realização de teste cutâneo de contato (patch test) no período do estudo e não uso de corticosteroide sistêmico ou outra medicação imunossupressora. Foram analisados idade, sexo, história de outros tipos de alergia e sensibilizantes utilizados. Os resultados do patch test foram lidos 48 horas e 72 horas após a colocação dos contensores.

Resultados: Foram incluídos 362 pacientes, entre o quais 60 idosos (idade maior ou igual a 60 anos) e 302 não idosos. Além da dermatite de contato foram observados outros tipos de alergias em 20 (33,3\%) dos idosos e em $113(37,4 \%)$ dos não idosos (p=0,54). Houve um total de 162 reações positivas ao patch test entre os idosos e 738 reações positivas entre os não idosos $(\mathrm{p}=0,24)$. Quanto às reações positivas fortes ocorreram $41(68,3 \%)$ entre os idosos e $156(51,6 \%)$ entre os não idosos $(\mathrm{p}=0,02)$. Somando-se reações positivas fortes e muito fortes, foram 44 $(73,3 \%)$ em idosos e $214(70,8 \%)$ em não idosos ( $\mathrm{p}=0,69)$. As substâncias mais sensibilizantes foram o sulfato de níquel (em 33,3\% dos idosos e $38 \%$ dos não idosos) e o perfume mix (em $25 \%$ dos idosos e $27,8 \%$ dos não idosos), sem diferença estatística entre os dois grupos etários. Conclusões: A taxa de positividade do patch test entre os pacientes com dermatite de contato foi alta, não havendo diferença entre idosos e não idosos, sendo o sulfato de níquel e o perfume mix as substâncias mais sensibilizantes. Na investigação etiológica da dermatite de contato deve-se pensar principalmente na possibilidade de sensibilização aos perfumes e ao níquel, independentemente da faixa etária.

DESCRITORES: dermatite de contato; idoso; perfumes; níquel; testes do emplastro.

\section{ABSTRACT}

Aims: To evaluate the prevalence of the main allergens found in allergic contact dermatitis among elderly and non-elderly patients.

Methods: Retrospective study of the medical records of patients clinically diagnosed with contact dermatitis treated from August 2000 to January 2012 at the Allergy Outpatient Clinic of Marília Medical School and at an Allergy and Dermatology private clinic in Marília, state of São Paulo, Brazil. The inclusion criteria were clinical diagnosis of contact dermatitis, patch test performed during the study period, and no treatment with systemic corticosteroids or any other immunosuppressants. Age, sex, history of other types of allergy and allergens used were assessed. The patch test results were read 48 hours and 72 hours after application of the test chambers.

Results: A total of 362 patients were included in the study: 60 elderly patients (aged 60 years or older) and 302 non-elderly patients. In addition to contact dermatitis, other types of allergies were observed in $20(33.3 \%)$ of elderly patients and in $113(37.4 \%)$ of non-elderly patients $(\mathrm{p}=0.54)$. There were 162 positive reactions on the patch test among elderly patients and 738 positive reactions among non-elderly patients $(\mathrm{p}=0.24)$. Strong positive reactions were present in $41(68.3 \%)$ elderly patients and in $156(51.6 \%)$ non-elderly patients $(\mathrm{p}=0.02)$. Strong and very strong positive reactions, when assessed together, were detected in $44(73.3 \%)$ elderly patients and in $214(70.8 \%)$ non-elderly patients $(\mathrm{p}=0.69$ ). The most common allergens were nickel sulfate (in $33.3 \%$ of elderly and $38 \%$ of non-elderly patients) and fragrance mix (in $25 \%$ of elderly and $27.8 \%$ of non-elderly patients), with no statistical difference between the groups.

Conclusions: There was a high rate of positive reactions on the patch test among patients with contact dermatitis, with no difference between elderly and non-elderly individuals, and the most common allergens were nickel sulfate and fragrance mix. Therefore, it is important that allergy to nickel and fragrances be included in the etiologic investigation of contact dermatitis, regardless of age.

KEY WORDS: dermatitis, contact; elderly; fragrances; nickel; patch tests. 
Abreviaturas: DC, dermatite de contato; DCA, dermatite de contato alérgica; DCI, dermatite de contato por irritante; FAMEMA, Faculdade de Medicina de Marília; GBEDC, Grupo Brasileiro de Estudo em Dermatite de Contato.

\section{INTRODUÇÃO}

A dermatite de contato (DC) é uma doença de alta prevalência, causada por agentes externos, que em contato com a pele desencadeiam uma reação inflamatória caracterizada clinicamente por lesões do tipo eczema, isto é, eritema, vesículas, exsudação, pápulas, descamação e liquenificação. A DC é responsável por grande parte das consultas aos especialistas das áreas da alergia e de dermatologia $[1,2]$.

A DC pode resultar de um irritante primário, denominando-se DC por irritante (DCI), a qual corresponde a aproximadamente $80 \%$ dos casos, ou pode ser uma DC alérgica (DCA), esta correspondendo a $20 \%$ dos casos. A DCA é consequência de uma reação de hipersensibilidade tipo IV, orquestrada por células $\mathrm{T}$ contra substâncias em geral de baixo peso molecular denominadas haptenos, enquanto a DCI é causada por ação direta de substâncias químicas irritantes na pele. Quando o desencadeamento da DCI está relacionado à exposição à luz podemos classificála como DC fototóxica ou DC fotoalérgica, sendo que no primeiro caso o mecanismo é semelhante ao da DCI, entretanto para que o contactante torne-se um irritante é necessária à exposição à luz. No segundo caso, o mecanismo fisiopatológico é semelhante ao da DCA, porém a exposição à luz é que transforma o contactante inerte em alergênico, desencadeando assim o processo imune [2-4].

O teste cutâneo de contato ou patch test é o método mais eficiente para confirmar o diagnóstico etiológico da DCA $[1,5,6]$. Ele pode sofrer influência de fatores genéticos, fatores relacionados à etnia e à idade do indivíduo [7-9]. As diretrizes britânicas sobre DC indicam o teste de contato para pacientes com erupções eczematosas persistentes, quando a DCA é suspeitada ou não pode ser descartada (nível de evidência II/ grau de recomendação A), cuja sensibilidade e especificidade estaria entre $70 \%$ e $80 \%$ [1], sendo que a Diretriz da Sociedade Europeia de Dermatite de Contato traz orientações excelentes e atualizadas sobre a utilização do patch test na investigação da DC [5].

Outro aspecto de interesse nos dias atuais é o aumento progressivo da população idosa e a existência de poucos estudos abordando as alergias nessa faixa etária, o que abre um campo novo e interessante para pesquisas, em particular no que diz respeito à investigação da DC. Assim, foi objetivo deste trabalho pesquisar os principais sensibilizantes relacionados à DC num grupo populacional brasileiro, comparando-se idosos com não idosos.

\section{MÉTODOS}

Foi realizado um estudo retrospectivo, em que foram avaliados os prontuários de pacientes com diagnóstico clínico de DC, atendidos no período de agosto de 2000 a janeiro de 2012, no Ambulatório de Alergia da Faculdade de Medicina de Marília (FAMEMA) e em clínica particular de Alergia e Dermatologia na cidade de Marília, localizada no interior do estado de São Paulo, Brasil. O projeto foi aprovado pelo Comitê de Ética em Pesquisa da FAMEMA (número 12624913.1.0000.5413) e o estudo foi supervisionado pelas Disciplinas de Alergia e Imunopatologia Clínica e de Dermatologia da mesma instituição. Por ser um estudo retrospectivo com pesquisa em prontuários, foi dispensada a assinatura de consentimento livre e esclarecido. As identidades dos pacientes foram preservadas, assim como todas as orientações da resolução 466/12 do Conselho Nacional de Saúde do Brasil.

Todos os pacientes com DC que realizaram patch test no período foram elegíveis para o estudo. $\mathrm{O}$ teste foi realizado por indicação clínica, e os resultados estavam descritos nos prontuários médicos. Verificaram-se os resultados do patch test com primeira leitura realizada 48 horas após a colocação dos contensores e a segunda leitura realizada 72 horas após a colocação. Também foram buscadas nos prontuários as medicações de uso rotineiro, verificando-se se haveria pacientes utilizando corticosteroides sistêmicos ou outros medicamentos imunossupressores, situações definidas como critérios de exclusão. Foram analisados os seguintes dados: idade, sexo, história de outros tipos de alergia e os sensibilizantes implicados. Foram considerados idosos os pacientes com 60 anos ou mais.

Foram testadas as seguintes substâncias produzidas pelo Alergofar Laboratório de Produtos Alergênicos (Rio de Janeiro), empresa brasileira registrada na Agência Nacional de Vigilância Sanitária: ácido benzoico, ácido bórico, ácido para-aminobenzoico, anilina, antraquinona, azocorante, bálsamo de peru, benzocaina, bicloreto de mercúrio, bicromato de potássio, butilfenol-paraterciário, carba-mix, cloranfenicol, 
cloreto de cobalto, clorexidina, colofônio, derivados imidazólicos, epóxi-resina, eosina, etilenodiamina, fenol, formaldeído, germall 115, hidroquinona, hipoclorito de sódio, irgasan, kathon CG, lanolina, látex, laurilsulfato de sódio, mercapto-mix, neomicina, nitrofurazona, parabeno-mix, parafenilenodiaminamix, perfume-mix, pirogalol, polietilenoglicol, prometazina, propilenoglicol, quinolina-mix, quaternium 15 , resorcina, sulfanilamida, sulfato de níquel, terebintina, tiuram-mix, timerosal, tolueno, viofórmio.

Antes da aplicação das substâncias a pele foi desengordurada com algodão embebido em álcool. As substâncias foram aplicadas no dorso do paciente por meio de contensores constituídos por fitas adesivas hipoalergênicas em formato retangular com discos de papel-filtro de $1,0 \mathrm{~cm}^{2}$ devidamente identificados. A quantidade aplicada de cada substância situou-se em torno de 15 microlitros para substâncias líquidas e $20 \mathrm{mg}$ para substâncias sólidas. Todos os pacientes foram orientados para não molhar o local, evitar coçar, evitar exercícios ou movimentos desnecessários. Ao serem removidas as fitas (após 48 horas), o paciente era orientado para que não lavasse o local até a próxima leitura de 72 horas.

Os critérios de leitura e classificação das intensidades das reações foram adotados com base nos definidos pelo Grupo Brasileiro de Estudo em Dermatite de Contato (GBEDC) [10]. Negativo: sem reação; duvidoso: eritema leve mal definido; positivo fraco: eritema definido, infiltração e pápula; positivo forte: eritema, infiltração, pápula e vesícula; positivo muito forte: eritema, infiltração, pápula, vesículas coalescentes formando bolha.

A análise estatística incluiu médias e medianas com desvios padrões ou intervalos interquartil para os dados quantitativos. Os dados qualitativos foram analisados por meio do teste do qui-quadrado, sendo as diferenças consideradas significativas quando $\mathrm{p} \leq 0,05$.

\section{RESULTADOS}

No período em estudo nenhum dos pacientes que realizaram o patch test estava fazendo uso de corticosteroide sistêmico ou qualquer outro tipo de imunossupressor. Dessa forma, foram incluídos no estudo todos os 362 pacientes com diagnóstico clínico de DC e submetidos ao patch test, entre o quais 60 idosos (idade maior ou igual a 60 anos) e 302 não idosos (idade menor ou igual a 59 anos). A média da idade encontrada nos idosos foi de $69,1 \pm 7,54$ anos, com mediana de 68,5 anos, idade mínima de
60 e máxima de 95 anos, predominando pacientes do sexo feminino $(66,7 \%)$. Nos não idosos a média de idade foi de 35,5 $\pm 12,5$ anos, mediana de 33,5 anos, idade mínima de 15 anos e máxima de 59 anos, predominando também pacientes do sexo feminino $(72,5 \%)$.

Além da DC foram observados outros tipos de alergias em $20(33,3 \%)$ dos idosos e em 113 $(37,4 \%)$ dos indivíduos não idosos $(\mathrm{p}=0,54)$. Quando analisadas cada uma destas alergias por faixa etária, encontramos as seguintes prevalências: rinite em $13,3 \%$ dos idosos e em $17,2 \%$ dos não idosos $(\mathrm{p}=0,45)$, asma em $1,6 \%$ dos idosos e em $3,9 \%$ dos não idosos $(\mathrm{p}=0,38)$, urticária crônica em $1,6 \%$ dos idosos e em $6,3 \%$ dos não idosos $(\mathrm{p}=0,15)$, dermatite atópica em $1,6 \%$ dos idosos e em $4,9 \%$ dos não idosos $(p=0,25)$ e reação adversa medicamentosa em $10 \%$ dos idosos e em $12,2 \%$ dos não idosos $(p=0,62)$. Portanto não houve diferença com significância estatística em nenhum dos processos alérgicos referidos nos prontuários, entre idosos e não idosos.

Descartando-se as reações negativas e duvidosas, houve um total de 162 reações positivas entre os idosos, portanto encontrando-se em média 2,7 reações positivas para cada idoso, sendo que entre os não idosos encontramos 738 reações positivas, perfazendo a média de 2,4 reações positivas para cada indivíduo, não havendo diferença com significância estatística entre os dois grupos $(p=0,24)$.

Para a comparação entre as faixas etárias em relação à positividade e intensidade das reações, foram consideradas todas as reações às substâncias testadas. Quanto ao número de pacientes com reações positivas fortes (considerando todas as substâncias testadas) encontramos $41(68,3 \%)$ entre os 60 idosos e $156(51,6 \%)$ entre os 302 não idosos $(p=0,02)$. Levando-se em consideração a somatória das reações positivas fortes e muito fortes, foram encontradas $44(73,3 \%)$ em idosos e $214(70,8 \%)$ em não idosos $(\mathrm{p}=0,69)$. As diferenças entre as duas faixas etárias na proporção de pacientes que apresentaram reações de outras intensidades também não foram estatisticamente significativas (Tabela 1).

Em relação às substâncias testadas, observamos que o sulfato de níquel e o perfume mix seguidos pelo timerosal, foram as substâncias mais sensibilizantes em ambos os grupos etários. Foram analisadas as prevalências de positividade para todas as substâncias testadas, não se encontrando diferenças com significância estatística sobre a prevalência de cada substância entre os grupos analisados (Tabela 2). 
Tabela 1. Proporção de pacientes com diagnóstico de dermatite de contato* que apresentaram reações positivas de diversas intensidades ao teste cutâneo de contato (patch test). Agosto de 2000 a janeiro de 2012, Ambulatório de Alergia da Faculdade de Medicina de Marília e clínica particular de Alergia e Dermatologia em Marília, SP.

\begin{tabular}{lcccc}
\hline & $\begin{array}{c}\text { Todos os pacientes } \\
\mathbf{n = 3 6 2}\end{array}$ & $\begin{array}{c}\text { Idosos } \\
\mathbf{n = 6 0}\end{array}$ & $\begin{array}{c}\text { Não idosos } \\
\mathbf{n = 3 0 2}\end{array}$ & $\mathbf{p}^{\dagger}$ \\
Reação duvidosa & $60(16,6 \%)$ & $8(13,3 \%)$ & $52(17,2 \%)$ & 0,46 \\
Reação positiva fraca & $147(40,6 \%)$ & $27(45 \%)$ & $120(39,7 \%)$ & 0,44 \\
Reação positiva forte & $197(54,4 \%)$ & $41(68,3 \%)$ & $156(51,6 \%)$ & $\mathbf{0 , 0 2}$ \\
Reação positiva muito forte & $137(37,8 \%)$ & $17(28,3 \%)$ & $120(39,7 \%)$ & 0,09 \\
\hline
\end{tabular}

* De acordo com os critérios do Grupo Brasileiro de Estudo em Dermatite de Contato [10].

+ Teste do qui quadrado. Comparação entre idosos e não idosos.

Observação: a soma das porcentagens em cada uma das colunas ultrapassa o valor de $100 \%$ pois reações de diferentes intensidades

foram observadas no mesmo paciente, dependendo da substância testada.

Tabela 2. Substâncias testadas por teste cutâneo de contato (patch test) com respectivas concentrações e porcentagens de sensibilização em 362 pacientes com diagnóstico de dermatite de contato. Agosto de 2000 a janeiro de 2012, Ambulatório de Alergia da Faculdade de Medicina de Marília e clínica particular de Alergia e Dermatologia em Marília, SP.

\begin{tabular}{|c|c|c|c|c|}
\hline \multirow{2}{*}{$\begin{array}{l}\text { Substâncias testadas } \\
\text { e concentrações }\end{array}$} & \multicolumn{3}{|c|}{ Porcentagens de sensibilização } & \multirow[b]{2}{*}{$\mathbf{p}^{*}$} \\
\hline & $\begin{array}{c}\text { Todos os pacientes } \\
n=362\end{array}$ & $\begin{array}{c}\text { Idosos } \\
\mathrm{n}=60\end{array}$ & $\begin{array}{c}\text { Não idosos } \\
n=302\end{array}$ & \\
\hline Sulfato de níquel 5\% & $36,7 \%$ & $33,3 \%$ & $38 \%$ & 0,48 \\
\hline Perfume mix $8 \%$ & $27 \%$ & $25 \%$ & $27,8 \%$ & 0,65 \\
\hline Timerosal 0,05\% & $23,6 \%$ & $20 \%$ & $24,8 \%$ & 0,42 \\
\hline Hidroquinona $1 \%$ & $14,1 \%$ & $13,3 \%$ & $14,5 \%$ & 0,80 \\
\hline Bálsamo de peru $25 \%$ & $12,2 \%$ & $11,6 \%$ & $12,6 \%$ & 0,84 \\
\hline Ácido benzoico 5\% & $11,9 \%$ & $11,6 \%$ & $12,3 \%$ & 0,89 \\
\hline Parafenilenodiamina mix $0,4 \%$ & $11,7 \%$ & $10 \%$ & $12,3 \%$ & 0,62 \\
\hline Derivados imidazólicos 7\% & $10,6 \%$ & $10 \%$ & $10,9 \%$ & 0,83 \\
\hline Parabeno-mix $15 \%$ & $9,2 \%$ & $10 \%$ & $9,3 \%$ & 0,85 \\
\hline Bicromato de potássio $0,5 \%$ & $8,2 \%$ & $6,7 \%$ & $9,3 \%$ & 0,51 \\
\hline Prometazina $1 \%$ & $7 \%$ & $5 \%$ & $7,6 \%$ & 0,47 \\
\hline Carba mix $3 \%$ & $6,8 \%$ & $5 \%$ & $7,3 \%$ & 0,52 \\
\hline Colofônio 20\% & $6,2 \%$ & $5 \%$ & $6,6 \%$ & 0,63 \\
\hline Tiuram mix $1 \%$ & $5,7 \%$ & $1,7 \%$ & $6,6 \%$ & 0,13 \\
\hline Benzocaína 5\% & $5,4 \%$ & $1,7 \%$ & $6,3 \%$ & 0,15 \\
\hline Epóxi resina 1\% & $5,2 \%$ & $1,7 \%$ & $6 \%$ & 0,17 \\
\hline Neomicina $20 \%$ & $4,6 \%$ & $1,7 \%$ & $5,3 \%$ & 0,22 \\
\hline Anilina 1\% & $4 \%$ & $1,7 \%$ & $4,6 \%$ & 0,29 \\
\hline Tolueno $0,5 \%$ & $4 \%$ & $1,7 \%$ & $5 \%$ & 0,29 \\
\hline Etilenodiamina $1 \%$ & $3,5 \%$ & $1,7 \%$ & $4 \%$ & 0,38 \\
\hline Lanolina 30\% & $3,5 \%$ & $1,7 \%$ & $4 \%$ & 0,38 \\
\hline Terebentina $10 \%$ & $3,2 \%$ & $1,7 \%$ & $3,6 \%$ & 0,43 \\
\hline Bicloreto de mercúrio 0,1\% & $3 \%$ & $1,7 \%$ & $3,3 \%$ & 0,49 \\
\hline Cloranfenicol 2\% & $3 \%$ & $1,7 \%$ & $3,3 \%$ & 0,49 \\
\hline Látex ao natural & $3 \%$ & $1,7 \%$ & $3,3 \%$ & 0,49 \\
\hline Sulfanilamida $5 \%$ & $3 \%$ & $1,7 \%$ & $3,3 \%$ & 0,49 \\
\hline Butilfenol para-terciário $1 \%$ & $2,7 \%$ & $1,7 \%$ & $3 \%$ & 0,57 \\
\hline Germal $1152 \%$ & $2,7 \%$ & $1,7 \%$ & $3 \%$ & 0,57 \\
\hline Kathon CG 0,5\% & $2,7 \%$ & $1,7 \%$ & $3 \%$ & 0,57 \\
\hline Nitrofurazona 1\% & $2,4 \%$ & $1,7 \%$ & $2,6 \%$ & 0,65 \\
\hline Viofórmio $6 \%$ & $2,4 \%$ & $1,7 \%$ & $2,6 \%$ & 0,65 \\
\hline Lauril sulfato de sódio 2\% & $2,2 \%$ & $1,7 \%$ & $2,3 \%$ & 0,75 \\
\hline Quinolina mix 6\% & $2,2 \%$ & $1,7 \%$ & $2,3 \%$ & 0,75 \\
\hline Propilenoglicol 5\% & $1,9 \%$ & $1,7 \%$ & $2 \%$ & 0,86 \\
\hline Antraquinona $2 \%$ & $1,9 \%$ & $1,7 \%$ & $2 \%$ & 0,86 \\
\hline Ácido para-amino-benzoico 10\% & $1,6 \%$ & $1,7 \%$ & $1,7 \%$ & 0,99 \\
\hline Cloreto de cobalto $2 \%$ & $1,4 \%$ & $1,7 \%$ & $1,3 \%$ & 0,83 \\
\hline Irgasan $1 \%$ & $1,4 \%$ & $1,7 \%$ & $1,3 \%$ & 0,83 \\
\hline Polietilenoglicol ao natural & $0,3 \%$ & $1,7 \%$ & $0 \%$ & Não calculado \\
\hline Ácido bórico 1\% & $1,4 \%$ & $0 \%$ & $1,7 \%$ & Não calculado \\
\hline Azocorante $1 \%$ & $1,4 \%$ & $0 \%$ & $1,7 \%$ & Não calculado \\
\hline
\end{tabular}


Tabela 2. (conclusão)

\begin{tabular}{lcccc}
\hline \multicolumn{1}{c}{$\begin{array}{c}\text { Substâncias testadas } \\
\text { e concentrações }\end{array}$} & $\begin{array}{c}\text { Podos os pacientes } \\
\mathbf{n = 3 6 2}\end{array}$ & $\begin{array}{c}\text { Porcentagens de sensibilização } \\
\text { Idosos } \\
\mathbf{n = 6 0}\end{array}$ & $\begin{array}{c}\text { Não idosos } \\
\mathbf{n = 3 0 2}\end{array}$ & p \\
Mercapto mix 2\% & $0,8 \%$ & $0 \%$ & $1 \%$ & Não calculado \\
Formaldeído 2\% & $0,5 \%$ & $0 \%$ & $0,6 \%$ & Não calculado \\
Hipoclorito de sódio 5\% & $0,5 \%$ & $0 \%$ & $0,6 \%$ & Não calculado \\
Eosina 50\% & $0,3 \%$ & $0 \%$ & $0,3 \%$ & Não calculado \\
Clorexedina 1\% & $0 \%$ & $0 \%$ & $0 \%$ & Não calculado \\
Fenol 0,5\% & $0 \%$ & $0 \%$ & $0 \%$ & Não calculado \\
Pirogalol 1\% & $0 \%$ & $0 \%$ & $0 \%$ & Não calculado \\
Resorcina 1\% & $0 \%$ & $0 \%$ & $0 \%$ & Não calculado \\
\hline
\end{tabular}

* Teste do qui-quadrado. Comparação entre idosos e não idosos.

\section{DISCUSSÃO}

A DC configura-se numa das principais queixas no âmbito das especialidades de alergia e dermatologia, representando uma importante causa de morbidade, incapacidade ocupacional e decréscimo da qualidade de vida das pessoas. A importância de seu monitoramento constitui um fato digno de a cada dois anos o grupo norte-americano de DC coletar e apresentar dados dos resultados do patch test de uma série padronizada de alérgenos [11-13].

O presente estudo envolveu 50 contactantes possivelmente presentes no ambiente dos pacientes avaliados, englobando portanto um número maior do que as 30 substâncias da bateria padrão preconizadas pelo GBEDC [10], portanto permitindo uma exploração mais ampla dos prováveis sensibilizantes e ou irritantes.

Demograficamente, a exemplo do observado no presente trabalho em que a amostragem de pacientes com DC foi composta predominantemente por mulheres, tanto no grupo de idosos quanto no de não idosos, outros estudos nacionais também verificaram maior número amostral de pacientes do sexo feminino com DC $[10,14,15]$. Em relação à idade, existem poucos trabalhos abordando os quadros alérgicos em idosos [16-29], sendo que dois estudos nacionais apontaram uma alta prevalência de manifestações cutâneas nessa faixa etária, entre as quais a DC [21] e o prurido, como sintoma isolado [22].

A eficiência da resposta imune pode sofrer um declínio com o avançar da idade, denominada imunossenescência [23]. Classicamente, a imunidade é classificada em dois braços inter-relacionados: a imunidade inata (natural), constituindo-se esta como a primeira linha de defesa do organismo, e a imunidade adaptativa (adquirida) responsável pela resposta mais elaborada e especializada do organismo, ambas afetadas pela imunossenescência. Durante a vida, o sistema imunológico sofre continuamente estímulos e mudanças morfológicas e funcionais, ocorrendo um pico de sua função na puberdade e um declínio gradual com o envelhecimento e a consequente involução do timo. Com o envelhecimento da pele, existe menor eficiência da barreira mecânica da mesma para possíveis agressores externos, assim como diminuição da hidratação, aumentando o risco para reações irritativas e penetração de potenciais sensibilizantes [9]. Há, também, um decréscimo na densidade de células de Langerhans, responsáveis pela apresentação de antígenos, o que pode colaborar para a menor resposta imune e, portanto, menor reatividade da pele do idoso. Por outro lado, a diminuição na taxa de renovação celular poderia implicar em maior tempo de exposição aos sensibilizantes, assim como em menor capacidade de recuperação frente aos agressores [8,24]. Outro aspecto a ser levado em consideração é o maior tempo e o grande número de agentes sensibilizantes aos quais o idoso esteve exposto ao longo de toda a sua vida. Portanto a reatividade da pele do paciente idoso poderia ser comparada a uma balança, a qual poderia inclinar-se para ambos os lados dependendo da força de dois aspectos principais: de um lado uma possível deficiência da resposta imunológica e, portanto, menor reatividade da pele, e por outro lado, maior exposição aos contactantes em virtude dos muitos anos vividos, que também estaria associada à menor taxa de renovação celular e ao ressecamento cutâneo, predispondo o indivíduo à maior ação das substâncias irritantes e sensibilizantes. Outros aspectos relevantes em relação aos idosos são as terapias para dermatites e úlceras de estase, assim como condições tipo incontinência urinária e fecal, que predispõem o idoso a DCI, assim como maior exposição às substâncias tópicas utilizadas nesses tratamentos [8].

No presente estudo, exceto pelo maior número de reações positivas fortes observadas no grupo de 
idosos, na grande maioria das avaliações, não foram encontrados resultados com diferença estatística entre idosos e não idosos. Também, o Grupo NorteAmericano de Dermatite de Contato, em análise retrospectiva realizada entre os anos de 1994 e 2008, observou que em idosos há maior probabilidade de ocorrerem testes positivos quando comparados às crianças, mas similares quando comparados aos adultos. Os autores concluíram que a frequência de positividade de reações alérgeno-específicas, conforme a idade, estariam mais relacionadas às exposições de cada grupo etário [25].

O presente estudo encontrou uma proporção significativamente maior de reações fortes nos idosos em relação aos não idosos. Levando-se em consideração, entretanto, a somatória das reações positivas fortes e muito fortes, as quais apresentam a melhor acurácia, com precisão entre 80 e 100\% [26], a diferença não foi significativa entre as duas faixas etárias. Um estudo brasileiro onde também foi estudada a DC em 80 idosos e em 581 adultos encontrou maior proporção de testes positivos nos idosos $(87,5 \%)$, com diferença estatística em relação aos adultos não idosos [27].

O principal sensibilizante encontrado em ambos os grupos analisados foi o sulfato de níquel, à semelhança do estudo nacional citado anteriormente [27] e estudos internacionais $[23,28,29]$, sendo que o segundo sensibilizante mais prevalente neste trabalho foi o perfume mix, contactante também altamente prevalente nos estudos anteriormente citados.

A DCA por níquel representa uma importante causa de perda da qualidade de vida, podendo levar a prejuízos ocupacionais e a um significante gasto com cuidados em saúde. Os quadros podem estar relacionados a doenças ocupacionais, embora a maioria seja relacionada a casos não ocupacionais. Vários materiais, incluindo metais como ouro e aço inoxidável, que compõem objetos de uso diário, contêm níquel em sua composição. A DCA por níquel ocorre quando esses objetos entram em contato com a pele, principalmente quando são corroídos pela sudorese, saliva ou outros fluídos corporais, liberando íons de níquel que atuam como hapteno, induzindo à sensibilização [30].

A quantidade de exposição ao níquel por área pode ser quantificada em $\mu \mathrm{g} / \mathrm{cm}^{2}$ e pode variar dependendo da quantidade de níquel liberada e do tempo de contato. A legislação Europeia estabeleceu o limite de $0,05 \%$ para a concentração de níquel em materiais que entram em contato com a pele e, para a quantia de liberação do níquel, o valor de $0,5 \mu \mathrm{g} / \mathrm{cm}^{2} / \mathrm{semana}$. Após essa regulamentação, ocorreu uma diminuição na prevalência da alergia ao níquel na Dinamarca e na Alemanha. O aço inoxidável e o ouro usualmente liberam menos que $0,5 \mu \mathrm{g} / \mathrm{cm}^{2} / \mathrm{semana}$ de níquel, mas outros materiais contendo níquel em geral liberam mais que essa quantidade, e, portanto, são uma importante causa de desencadeamento e agravamento da DCA por níquel [30,31].

Essa ocorrência é comum com bijuterias, sendo possivelmente uma das causas da maior prevalência de DCA nas mulheres. Os brincos constituem uma das principais causas de desencadeamento da DCA por níquel. Além das orelhas, o pescoço e as pálpebras são locais frequentes de sensibilização em virtude do uso de colares e de esmalte de unhas contendo níquel, por contato dos dedos com o pescoço e a região dos olhos. $\mathrm{O}$ níquel também pode ser encontrado em produtos de maquiagem, tintura de cabelo, tintura de tecidos e nos mais diversos objetos metálicos, como pulseiras, relógios de pulso, botões, moedas e peças do vestuário [30-33].

No aspecto da exposição ocupacional o níquel também se destaca, pois um estudo brasileiro mostrou a exposição ocupacional em 39\% de 404 pacientes com reação positiva a algum dos três metais estudados: níquel, cobalto e cromo, destacando-se o níquel como o mais sensibilizante entre os três, de maneira isolada ou associada [32]. Um estudo britânico encontrou a sensibilização ao níquel em $12 \%$ de um total de 1190 casos de DC ocupacional [33]. Habitualmente a sensibilização ocorre por exposição não ocupacional, com agravamento após a exposição no trabalho; porém ocasionalmente a sensibilização ocorre no próprio local de trabalho, principalmente com atividades em ambientes úmidos concomitantes ao contato com o níquel. Em geral, esses trabalhadores desenvolvem eczemas crônicos, principalmente nas mãos [31].

Em relação às fragrâncias, sabe-se que constituem a causa mais comum das DC por cosméticos, sendo os componentes da fragrância mix assim constituídos: aldeído cinâmico, álcool cinâmico, álcool alfa-amilcinâmico, geraniol, eugenol, isoeugenol, oak moss absoluto e hidroxicitronelal. As reações podem ocorrer pelo contato com perfumes, cosméticos, sabonetes, detergentes, medicações, papeis e lenços de higiene, ou mesmo itens perfumados de outras pessoas [34].

A DC aos perfumes pode se apresentar de forma generalizada ou localizada, como nas mãos, face, pescoço, tronco, axilas e pernas, podendo associar-se ou não ao risco ocupacional. Na questão ocupacional a DC foi encontrada principalmente em fisioterapeutas, massagistas, enfermeiras de idosos e outros profissionais que têm contato direto com o corpo de outras pessoas [35]. 
Um estudo multicêntrico, abrangendo Europa, Estados Unidos e Japão, cujo objetivo foi identificar novos possíveis componentes químicos (outras fragrâncias) para a pesquisa da DC a perfumes, verificou que a reação à fragrância mix ocorreu em $76 \%$ dos pesquisados. Naquele estudo a alergia por perfumes acometeu preferencialmente mulheres, em face e mãos, sendo que muitos pacientes relataram história pessoal pregressa de rinite, asma ou dermatite atópica [36].

O uso de perfumes em áreas pré-lesadas por eczema parece favorecer novas sensibilizações; também, caso as fragrâncias estejam contidas em misturas com irritantes como sabões, detergentes ou outros possíveis irritantes primários, os mesmos poderiam atuar como coadjuvantes na precipitação da alergia, assim como a aplicação de desodorantes ou outros perfumes em área recentemente depilada e irritada, como nas axilas [37].

O timerosal ocupou o terceiro lugar em relação à prevalência das substâncias mais sensibilizantes em ambos os grupos, não havendo diferença com significância estatística entre os grupos. O timerosal foi um antisséptico muito utilizado no passado em medicamentos, principalmente em vacinas e colírios assim como em cosméticos, estando atualmente proibida sua comercialização no Brasil.

Quanto às limitações deste estudo, sem dúvida a principal é por se tratar de um estudo retrospectivo, o que pode ocasionar falta de precisão em relação aos dados pregressos já anotados. Também o fato da segunda leitura ter sido realizada com 72 horas e não com 96 horas, o que teoricamente poderia possibilitar um número maior de falsos negativos. Entretanto os cuidados locais exigidos, como não poder molhar o local, o que impediria o paciente de tomar banho por um tempo maior ( 96 horas ao invés de 72 horas), dificulta esta forma de abordagem em região de clima tropical, além do que, a Diretriz da Sociedade Europeia de Dermatite de Contato [5] admite que a segunda leitura seja feita tanto em 72 horas como em 96 horas.

Concluímos que a taxa de positividade do patch test entre os pacientes testados foi alta, não havendo diferença entre idosos e não idosos, sendo o sulfato de níquel e o perfume mix, seguidos pelo timerosal, as substâncias mais sensibilizantes nesta amostra. Consideramos serem necessários mais estudos abordando este assunto, particularmente na população idosa.

\section{NOTA}

Declaração de conflitos de interesse

Os autores declaram não haver conflitos de interesse relevantes ao conteúdo deste estudo.

\section{REFERÊNCIAS}

1. Bourke J, Coulson I, English J. Guidelines for the management of contact dermatitis: an update. Br J Dermatol. 2009;160:946-54. http://dx.doi.org/10.1111/j.1365-2133.2009.09106.x

2. Motta AA, Aun MV, Kalil J, Giavina-Bianchi P. Dermatite de contato. Rev Bras Alerg Imunopatol.2011;34(3):73-82.

3. Fonacier LS, Dreskin SC, Leung DYM. Allergic skin disease. J Allergy Clin Immunol. 2010;125:S138-49. http://dx.doi.org/10.1016/j. jaci.2009.05.039

4. Martins LEAM, Reis VMS. Imunopatologia da dermatite de contato alérgica. An Bras Dermatol. 2011;86(3):419-33. http://dx.doi. org/10.1590/S0365-05962011000300001

5. Johansen JD, Aalto-Korte K, Agner T, Andersen KE, Bircher A, Bruze M, Cannavó A, Giménez-Arnau A, Gonçalo M, Goossens A, John SM, Lidén C, Lindberg M, Mahler V, Matura M, Rustemeyer T, Serup J, Spiewak R, Thyssen JP, Vigan M, White IR, Wilkinson M, Uter W. European Society of Contact Dermatitis guideline for diagnostic patch testing - recommendations on best practice. Contact Dermatitis. 2015 Oct; 73(4):195-221. http://dx.doi.org/10.1111/cod.12432

6. Duarte I, Lazzarini R, Buense R, Pires MC. Dermatite de contato. An Bras Dermatol. 2000;75(5):529-48.

7. Deleo VA, Taylor SC, Belsito DV, Fowler JF Jr, Fransway AF, Maibach HI, Marks JG Jr, Mathias CG, Nethercott JR, Pratt MD, Reitschel RR, Sherertz EF, Storrs FJ, Taylor JS. The effect of race and ethnicity on patch test results. J Am Acad Dermatol. 2002 Feb;46(2 Suppl Understanding):S107-12.

8. Prakash AV, Davis MDP. Contact dermatites in older adults - a review of literature. Am J Clin Dermatol.2010;11(6):373-81. http://dx.doi. org/10.2165/11319290-000000000-00000

9. Seyfarth F, Schliemann S, Antonov D, Elsner P. Dry skin barrier function and irritant contact dermatites in the elderly. Clin Dermatol. 2011 Jan-Feb;29(1):31-6. http://dx.doi.org/10.1016/j.clindermatol.2010.07.004

10. Grupo Brasileiro de Estudo em Dermatite de Contato; Sociedade Brasileira de Dermatologia. Departamento Especializado de Alergia. Estudo multicêntrico para elaboração de uma bateria-padrão brasileira de teste de contato. An Bras Dermatol. 2000;75(2):147-56.

11. Zug KA, Warshaw EM, Fowler JF Jr, Maibach HI, Belsito DL, Pratt MD, Sasseville D, Storrs FJ, Taylor JS, Mathias CG, Deleo VA, Rietschel RL, Marks J. Patch-testresults of the North American Contact Dermatitis Group 2005-2006. Dermatitis. 2009 MayJune;20(3):149-60. 
12. Fransway AF, Zug KA, Belsito DV, Deleo VA, Fowler JF Jr, Maibach HI, Marks JG, Mathias CG, Pratt MD, Rietschel RL, Sasseville D, Storrs FJ, Taylor JS, Warshaw EM, Dekoven J, Zirwas M. North American Contact Dermatitis Group patch test results for 2007-2008. Dermatitis. 2013 Jan-Feb;24(1):10-21. http://dx.doi.org/10.1097/DER.0b013e318277ca50

13. Warshaw EM, Belsito DV, Taylor JS, Sasseville D, DeKoven JG, Zirwas MJ, Fransway AF, Mathias CG, Zug KA, DeLeo VA, Fowler JF Jr, Marks JG, Pratt MD, Storrs FJ, Maibach HI. Dermatitis. 2013 Mar-Apr;24(2):50-9. http://dx.doi.org/10.1097/DER.0b013e3182819c51

14. Rodrigues DF, Neves DR, Pinto JM, Alves MFF, Fulgêncio ACF. Results of patch-tests from Santa Casa de Belo Horizonte Dermatology Clinic, Belo Horizonte, Brazil, from 2003 to 2010. An Bras Dermatol. 2012;87(5):800-3. http://dx.doi.org/10.1590/S0365-05962012000500028

15. Minelli L, Swenson AM. Estudo de 70 casos de eczema de contato: alérgenos observados. Rev Bras Alerg Imunopatol.1997;20(5):173-8.

16. Bendkowski B. Allergic diseases in the elderly. Gen Practit. 1968;16:208-14

17. Wöhrl S, Stingl G. Underestimation of allergies in elderly patients. Lancet. 2004;363:249. http://dx.doi.org/10.1016/S0140-6736(03)15349-4

18. Becerril AM, Vázquez MCL, Angeles GU, Alvarado MLE, Vilchis GE. Prevalencia de enfermidades alérgicas em adultos mayores. Ver Alerg Mex. 2008;55(3):85-91.

19. Ventura MT, D'Amato A, Giannini M, Carretta A, Tummolo RA, Buquicchio R. Incidence of allergic diseases in na elderly population. Immunopharmacol Immunotoxicol. 2010;32(1):165-70. http://dx.doi.org/10.3109/08923970903213735

20. Cardona V, Guilarte M, Luengo O, Labrador-Horrillo M, Sala-Cunill A, Garriga T. Allergic diseases in the elderly. Clin Transl Allergy. 2011;1(1):e11. http://dx.doi.org/10.1186/2045-7022-1-11

21. Alves LDS, Calamita ABP, Calamita Z. Estudo comparativo da prevalência de alergias entre idosos e não idosos. Braz J Allergy Immunol. 2014:2(2):75-80.

22. Alves LDS, Calamita Z. Sintomas de alergia em idosos atendidos em um ambulatório de geriatria. Sci Med. 2014;24(3):269-73. http://dx.doi.org/10.15448/1980-6108.2014.3.16326

23. Balato A, Balato N, Di Constanzo L, Ayala F. Contact sensitization in the elderly. Clin Dermatol. 2011;29:24-30. http://dx.doi.org/10.1016/j. clindermatol.2010.07.003

24. Accursio CSC. Alterações da pele na Terceira idade. Rev Bras Med. 2001;58(9):646-58.

25. Warshaw EM, Raju SI, Fowler JF Jr, Maibach HI, Belsito DV, Zug KA, Rietschel RL, Taylor JS, Mathias CG, Fransway AF, DeLeo VA, Marks JG Jr, Storrs FJ, Pratt MD, Sasseville D; North American Contact Dermatitis Group, 1994-2008. Positive patch test reactions in older individuals: retrospective analysis from the North American Contact Dermatitis Group, 1994-2008. J Am Acad Dermatol. 2012 Feb;66(2):229-40. http://dx.doi.org/10.1016/j.jaad.2010.12.022

26. Ali SA. Testes epicutâneos, testes de contato ou "patch tests" porque usar e como usar. Rev Bras Alerg Imunopatol. 1993;16(5):192-7.

27. Duarte I, Kobata C, Lazzarini R. Dermatite de contato em idosos. An Bras Dermatol. 2007;82(2):135-40. http://dx.doi.org/10.1590/S036505962007000200004

28. Önder M, Öztas MO. Contact dermatitis in the elderly. Contact Dermatitis. 2003;48(4):232-3. http://dx.doi.org/10.1034/j.16000536.2003.00060.x

29. Tosti A, Pazzaglia M, Silvani S, Delorenzi F. The espectrum of allergic contact dermatites in the elderly. Contact Dermatitis. 2004;50(6): 379-81. http://dx.doi.org/10.1111/j.0105-1873.2004.0350g.x

30. Torres F, Graças M, Melo M, Tosti A. Management of contact dermatitis due to nickel allergy: an update. Clin Cosmet Investig Dermatol. 2009;2:39-48.

31. Kim YY, Kim MY, Park YM, Kim HO, Koh CS, Lee HK. Evaluating the nickel content in metal alloys and the threshold for nickel-induced allergic contact dermatitis. J Korean Med Sci. 2008;23:315-9. http://dx.doi.org/10.3346/jkms.2008.23.2.315

32. Duarte I, Amorim JR, Perázzio EF, Schmitz Junior R. Metal contact dermatitis: prevalence of sensitization to nickel, cobalt and chromium. An Bras Dermatol. 2005;80(2):137-42. http://dx.doi.org/10.1590/S0365-05962005000200003

33. Shum KW, Meyer JD, Chen Y, Cherry N, Gawkrodger DJ. Occupational contact dermatitis to nickel: experience of the British dermatologists (EPIDERM) and occupational physicians (OPRA) surveillance schemes. Occup Environ Med. 2003;60:954-7. http://dx.doi.org/10.1136/ oem.60.12.954

34. Scheinman PL. Allergic contact dermatitis to fragrance: a review. Am J Contact Dermat. 1996;7(2):65-76.

35. Uter W, Schnuch A, Geier J, Pfahlberg A, Gefeller O. Association between occupation and contact allergy to the fragrance mix: a multifactorial analysis of national surveillance data. Occup Environ Med. 2001;58:392-8. http://dx.doi.org/10.1136/oem.58.6.392

36. Larsen W, Nakayama H, Fischer T, Elsner P, Frosch P, Burrows D, Jordan W, Shaw S, Wilkinson J, Marks J, Sugawara M, Nethercott M, Nethercott J. Fragrance contact dermatitis - a worldwide multicenter investigation (part III). Contact Dermatitis. 2002;46(3):141-4. http://dx.doi.org/10.1034/j.1600-0536.2002.460302.x

37. Guin JD, Berry VK. Perfume sensitivity in adult females: a study of contact sensitivity to a perfume mix in two groups of student nurses. J Am Acad Dermatol. 1980;3:299-302. http://dx.doi.org/10.1016/S0190-9622(80)80193-9 C 